\title{
AC Electric-Field-Induced Fluid Flow in Microelectrode Structures: Scaling Laws
}

\author{
Antonio Castellanos ${ }^{1}$, Antonio Ramos ${ }^{1}$, Antonio González ${ }^{2}$, \\ Hywel Morgan ${ }^{3}$, Nicolas Green ${ }^{3}$
}

${ }^{1}$ Dept. Electrónica y Electromagnetismo, Univ.de Sevilla, Facultad de Fisica, 41012 Sevilla, SPAIN.
2Dept. Física Aplicada III, Universidad de Sevilla, E.S. Ingenieros 41092 Sevilla, SPAIN.
${ }^{3}$ Dept. Electronic and Electrical Engineering, Univ. of Glasgow, Rankine Bldg, Glasgow G12 8QQ, UK.

\begin{abstract}
The motion of polarizable bioparticles under the action of non-uniform ac electric fields is known as dielectrophoresis. Because submicrometre particles are subjected to Brownian motion, high electric fields are needed to manipulate them. However, these high electric fields give rise to fluid motion, which in tum results in a viscous drag on the particles. The electric fields generate heat, leading to gradients in conductivity, permittivity, and mass density in the fluid. These gradients give rise to electrothermal forces and buoyancy. Also non-uniform ac electric fields produce forces on the induced charges in the diffuse double layer on the electrodes, that results in fluid motion. Here we characterize these flows and their domain of influence.
\end{abstract}

\section{INTRODUCTION}

The use of ac dielectrophoretic forces to control and manipulate particles of micron size in microelectrode structures is a well-established technique [1]. In particular, the dielectrophoretic manipulation of bioparticles such as viruses, cells, DNA is of special interest. It is to be expected that as we downsize the system in order to manipulate sub-micron particles Brownian motion will impose some limitation to this process. However the experiments carried out to set the precise limit to this miniaturisation revealed that the liquid was put in motion, and that this motion was a limiting factor that could be far more important than Brownian motion. It soon became clear that due to the high intensity electric fields involved and the small size of the system it appeared that Joule heating could be important enough to give rise to buoyancy forces and to forces of electrical origin induced by the variation of conductivity and permittivity in the suspending aqueous solution (electrothermal forces) [2]],[3]. In addition, the geometry of the system imposed a non-zero tangential electric field at the electrode double layers that put the liquid into steady motion. This motion was termed ACelectroosmosis as it is akin to electro-osmotic flow[4] [5].

In order to manipulate the particles it is useful to know how the flows scale with the size of the system, the shape of the electrodes, the magnitude and frequency of the applied ac electric field, and with the conductivity of the solution. Here we present the quantitative results obtained for a simplified system of two infinitely half-plane electrodes a distance $l$ apart, in order to gain a first understanding of these scaling laws. With due precautions these results can be generalised to more complicated microelectrodes shapes by taking into account that different zones may have different characteristic length scales.

\section{NATURE OF THE PROBLEM}

Bioparticles, such as viruses $(0.05-0.1 \mu \mathrm{m})$, bacteria $(0.5$ $1.5 \mu \mathrm{m})$, red blood cells $(5 \mu \mathrm{m})$ or lymphocytes $(5-8 \mu \mathrm{m})$ are usually in aqueous saline solutions with a conductivity that ranges between $10^{-4}$ and $10^{-1} \mathrm{~S} / \mathrm{m}$. Typical system lengths of microelectrodes (interelectrode gaps) used in the dielectrophoretic manipulation of bioparticles vary from 1 to 500 microns. Applied signals to these electrode structures range from 0 to 20 Volts giving rise to electric fields that can be as high as $5 \times 10^{6} \mathrm{~V} / \mathrm{m}$. These applied signals have frequencies that range between $10^{2}$ to $10^{7} \mathrm{~Hz}$.

In our experiments, we have used a simple electrode design consisting of two coplanar rectangular electrodes $2 \mathrm{~mm}$ long and $500 \mu \mathrm{m}$ wide, with parallel edges separated by a $25 \mu \mathrm{m}$ gap mounted on a glass substrate. Because the gap is small compared to the length and width of the electrodes most of the relevant physics can be considered two-dimensional [5],[6] , and the analysis is restricted to the $2 \mathrm{D}$ cross-section. In order to obtain some numbers, the electric field lines between electrodes are going to be considered semi-circular when needed. In this case, the electric field is $\mathbf{E}=V / \pi r \mathbf{u}_{\theta}$, where $V$ is the voltage amplitude of the applied signal and $r$ is the distance to the centre of the gap. The solutions for this simple system can give indications about the relative order of magnitude of the different forces involved, and the different regions of validity of the approximations made.

\section{Electrical equations}

The electromagnetic field in the bulk is governed by Maxwell's equations. Nevertheless, we can neglect the magnetic effects against the electrical ones for microelectrode structures since the energy stored in the magnetic field is much smaller than the electrical energy. Under these conditions the electromagnetic equations reduce to the quasielectrostatic limit [7].

For saline solutions the convection current can be neglected in front of the ohmic current. Assuming that conductivity $\sigma$ and permittivity $\varepsilon$ are independent on time, the equations that govern $\mathrm{AC}$ fields of frequency $\omega$ are 


$$
\nabla \times \mathbf{E}=0, \quad \nabla \cdot((\sigma+i \omega \varepsilon) \mathbf{E})=0
$$

In many cases, the gradients in permittivity and conductivity are small [3][6], and we can perform an expansion in the field as $\mathbf{E}=\mathbf{E}_{0}+\mathbf{E}_{1}\left(\left|\mathbf{E}_{1}\right|<<\left|\mathbf{E}_{0}\right|\right)$, where the fields verify the equations

$$
\nabla \cdot \mathbf{E}_{0}=0, \quad \quad \nabla \cdot \mathbf{E}_{1}+\left(\frac{\nabla \sigma+i \omega \nabla \varepsilon}{\sigma+i \omega \varepsilon}\right) \mathbf{E}_{0}=0
$$

The charge density at order one can be approximated as

$$
\rho_{1}=\varepsilon \nabla \cdot \mathbf{E}_{1}+\nabla \varepsilon \cdot \mathbf{E}_{0}=\left(\frac{\sigma \nabla \varepsilon-\varepsilon \nabla \sigma}{\sigma+i \omega \varepsilon}\right) \mathbf{E}_{0}
$$

We will be mainly concerned with the zero order field, whose electric potential verifies Laplace's equation.

\section{Mechanical equations}

The liquid motion is governed by the Navier-Stokes equations for an incompressible fluid. We are interested in the timeaveraged flow. The inertial terms are negligible, as for microsystems, the Reynolds number is usually very small $(v<100 \mu \mathrm{m} / \mathrm{s}, k 100 \mu \mathrm{m})$. Since the mass density of the fluid is almost homogeneous, the Boussineq approximation is valid. Therefore Navier-Stokes equations reduce to

$$
\nabla \cdot \mathbf{v}=0, \quad 0=-\nabla p+\eta \nabla^{2} \mathbf{v}+\left\langle\mathbf{f}_{E}\right\rangle+\Delta \rho_{m} \mathbf{g}
$$

As stated in this equation the fluid motion is caused by the joint action of gravity and electrical forces. The time average electrical force is given by [3][6]

$$
\left\langle\mathbf{f}_{E}\right\rangle=\frac{1}{2} \operatorname{Re}\left(\left(\left(\frac{\sigma \nabla \varepsilon-\varepsilon \nabla \sigma}{\sigma+i \omega \varepsilon}\right) \mathbf{E}_{0}\right) \mathbf{E}_{0}^{*}\right)-\frac{1}{4} \mathbf{E}_{0} \cdot \mathbf{E}_{0}^{*} \nabla \varepsilon
$$

If $\omega$ is much greater than $\sigma / \varepsilon$, the second term, the dielectric one, dominates. While if $\omega \ll \sigma / \varepsilon$, the first term, the Coulomb one, tends to dominate because relative variations in conductivity $(\Delta \sigma / \sigma)$ are usually much greater than relative variations in permittivity $(\Delta \varepsilon / \varepsilon)$. This is, in fact, the case for variations due to gradients in temperature, where $(1 / \sigma)(\partial \sigma / \partial \mathrm{T}) \gg>(1 / \varepsilon)(\partial \varepsilon / \partial \mathrm{T})$ in electrolytes.

\section{Temperature equation}

Together with the electrical and mechanical equations, we have the equation for the temperature. The heat convection is small compared to heat diffusion in microsystems, and the time-averaged temperature equation reduces to Poisson's equation, with the Joule heating as heat source

$$
\nabla^{2} T=-\frac{\sigma}{k}\left\langle E^{2}\right\rangle
$$

\section{Boundary conditions}

\section{Electric potential}

The electric potential at the surface of the electrodes is fixed by the source. Nevertheless, this is not necessarily the most suitable boundary condition to describe the behaviour of the potential near the electrodes. The reason lies in the presence of a double layer between the metallic surface and the electrolyte, which in our case is of nanometer size. The electrical behaviour of this double layer can be modelled. theoretically as a capacitor between the electrode and the electrolyte. The corresponding boundary condition expresses that the current that goes into this capacitor equals the increase in the stored charge [8][9]

$$
\phi-\frac{\sigma}{i \omega C} \frac{\partial \phi}{\partial n}=V
$$

For the interface electrolyte/glass the boundary condition can be approximated by

$$
\text { (8) } \frac{\partial \phi}{\partial n}=0
$$

For the upper boundary, we can consider that the points are at the infinity and the electric field and potential vanish at that boundary.

\section{Velocity}

In any of the rigid boundaries, the normal velocity vanishes. The tangential velocity, however, can be different from zero on the electrodes. This is due to the presence of the double layer at the electrolyte/electrode interface. The effect of the tangential AC field on the oscillating charges can be modelled as surface stresses that result in a slip velocity given by a generalisation of the Smoluchowsky formula. The timeaverage expression for the slip velocity is given by [8][9]

$$
u=\frac{1}{2} \frac{\varepsilon}{\eta} \Lambda \operatorname{Re}\left[(\Delta \phi) E_{t}^{*}\right]=-\frac{1}{4} \frac{\varepsilon}{\eta} \Lambda \frac{\partial}{\partial x}|\Delta \phi|^{2}
$$

where $\Delta \phi$ represents the voltage drop across the double layer and $E_{t}$ the tangential field just outside the double layer. The parameter $\Lambda$ is given by

$$
\Lambda=\frac{C_{S}}{C_{S}+C_{D}}
$$

where $C_{S}$ and $C_{D}$ are the capacitances per unit of area of the Stern and diffuse layers, respectively. For the rest of the lower boundary the effect of the double layer is negligible and the tangential velocity vanishes due to viscous friction [9].

\section{Temperature}

In the experiments in microelectrodes the boundary conditions for the temperature field are given by the surroundings and it can be very different between experiments. In order to make some calculations, we will consider that the electrodes are at room temperature. Also, the upper and lateral boundaries are going to be considered at room temperature placed at a distance of the order of the total size of the electrodes. In a previous paper [6] we have discussed the differences that obtained when different boundary conditions are used.

\section{FLUID FLOWS: SCALING LAWS}

\section{Joule-heating-induced electrothermal fluid flow}

The analytical solution assuming that the electrodes are at constant temperature [3] is

$$
\Delta T=\frac{\sigma V^{2}}{2 k}\left(\frac{\theta}{\pi}-\frac{\theta^{2}}{\pi^{2}}\right)
$$

where $\theta$ is the polar angle (at the electrodes $\theta=0, \theta=\pi$ ). This solution gives a maximum temperature increment of $\Delta T=\sigma V^{2} / 8 k$. This temperature field generates gradients 
in $\sigma$ and $\varepsilon$, giving rise to an electrical body force. The expression of the volume force for an electric field like $\mathbf{E}=(V / \pi r) \mathbf{u}_{\theta}$ is

$$
\mathbf{f}_{E}=-M \frac{\varepsilon \sigma V^{4}}{8 k(\pi r)^{3} T}\left(1-\frac{2 \theta}{\pi}\right) \mathbf{u}_{\theta}
$$

where $M$ is a dimensionless factor:

$$
M=\frac{\frac{T}{\sigma} \frac{\partial \sigma}{\partial T}-\frac{T}{\varepsilon} \frac{\partial \varepsilon}{\partial T}}{1+(\omega \varepsilon / \sigma)^{2}}+\frac{1}{2} \frac{T}{\varepsilon} \frac{\partial \varepsilon}{\partial T}
$$

At $T=300 \mathrm{~K}, M=6.6$ for $\omega \varepsilon / \sigma<<1$, and $M=-0.6$ for $\omega \varepsilon / \sigma \gg 1$. With this expression for the force an analytical solution for the velocity can be obtained. We can write estimations of the maximum velocity for the cases $\omega \varepsilon / \sigma \ll<1$ and $\omega \varepsilon / \sigma>>1$

$$
v_{\max } \sim\left\{\begin{array}{cc}
5 \times 10^{-4} \frac{\varepsilon \sigma V^{4}}{k \eta r}\left|\frac{1}{\sigma} \frac{\partial \sigma}{\partial T}\right| & \omega \ll \sigma / \varepsilon \\
2.5 \times 10^{-4} \frac{\varepsilon \sigma V^{4}}{k \eta r}\left|\frac{1}{\varepsilon} \frac{\partial \varepsilon}{\partial T}\right| & \omega \gg>\sigma / \varepsilon
\end{array}\right.
$$

Computations using the finite element method for our microelectrode system gave estimations of the maximum velocity [6] of the same order as the previous expression when $r$ is around $50 \mu \mathrm{m}$. That was the typical size of the convective roll in the computations. This is a value between the interelectrode gap length $(25 \mu \mathrm{m})$ and the height of the upper boundary $(200 \mu \mathrm{m})$.

\section{Joule heating induced buoyancy}

The gravitational body force generated by a temperature field as given in (11) is

$$
\mathbf{f}_{g}=\left(\frac{\partial \rho_{m}}{\partial T}\right) \frac{\sigma V^{2}}{2 k}\left(\frac{\theta}{\pi}-\frac{\theta^{2}}{\pi^{2}}\right) \mathbf{g}
$$

An order of magnitude estimate of the ratio between the electrical and gravitational forces gives for the case $\omega \varepsilon / \sigma<<1$

$$
\frac{f_{g}}{f_{E}} \sim \frac{\left(\frac{\partial \rho_{m}}{\partial T}\right)(\pi r)^{3} g}{\frac{1}{\sigma}\left(\frac{\partial \sigma}{\partial T}\right) \varepsilon V^{2}}
$$

For values in microelectrodes of $r=25 \mu \mathrm{m}, V=10$ volts and the other parameters those for water the ratio is $f_{g} / f_{E} \sim 7 \times 10^{-4}$ and the gravitational force is negligible in front of the electrical force for microelectrodes. As the typical size of the system is increased the order of magnitude of the gravitational force becomes greater than the electrical force. The transition is for $r$ around $300 \mu \mathrm{m}$. Finite element. computations confirm that a transition between buoyancy and electrical convection is obtained for typical size $r=300 \mu \mathrm{m}$. These computations give a typical velocity due to buoyancy of the form

$$
v_{\max } \sim 2 \times 10^{-2}\left(\frac{\partial \rho_{m}}{\partial T}\right) \frac{\sigma V^{2} g r^{2}}{k \eta}
$$

\section{AC electroosmosis}

Recent experimental work has shown that the application of an $\mathrm{AC}$ electric field to a pair of co-planar microelectrodes generates a steady (non-zero time averaged) fluid flow with a velocity which depends both on the applied potential and frequency [4][5][9]. A simple model based on an array of resistor-capacitor circuits can give reasonable values of the velocity. This model consisted of semicircular resistors bridging one electrode to the other, terminated at either end on an electrode by a capacitor representing the electrical double layer [4]. This model was further refined by taking into account the full linear electrokinetic equations for the double layer and the electrolyte [8]. Using this simple model $\Delta \phi$ is given by

$$
\Delta \phi=\frac{V}{2+i \pi C \omega r / \sigma}
$$

and the slip velocity is then

$$
u=\Lambda \frac{\varepsilon V^{2}}{8 \eta r} \frac{\Omega^{2}}{\left(1+\Omega^{2}\right)^{2}}, \quad \Omega=\frac{\omega C \pi r}{\sigma}
$$

A typical value of $C$ is $\varepsilon / \lambda_{D}$, the surface capacitance of the diffuse double layer in the Debye-Huckel model. Experimentally, $\Lambda$ is around 0.25 for our electrodes made of titanium and a medium conductivity around $0.002 \mathrm{Sm}^{-1}$. For an ideal double layer made of a Stern layer and a diffuse layer, the parameter $\Lambda$ decreases with conductivity, since the capacitance of the diffuse layer increases with conductivity. The velocity as a function of frequency has a maximum, and tends to zero for frequency going to zero or infinity. The maximum velocity is obtained for $\Omega=1$, that is, for angular frequency $\omega_{0}=\sigma /(C \pi r)$. Taking $C=\varepsilon / \lambda_{D}$, the angular frequency for maximum is $\omega_{0}=\left(\sigma \lambda_{D}\right) /(\varepsilon \pi r)$. This is several orders of magnitude smaller than the charge relaxation frequency of the liquid $\omega=\sigma / \varepsilon$.

\section{RESULTS AND DISCUSSION}

For the following results we will use typical physical quantities for aqueous saline solutions at a temperature of $T=298 \mathrm{~K}: \rho_{m}=10^{3} \mathrm{~kg} \mathrm{~m}^{-3}, \eta=10^{-3} \mathrm{~kg} \mathrm{~m}^{-2} \mathrm{~s}^{-1}, \varepsilon=7 \times 10^{-10} \mathrm{Fm}^{-1}$, $(1 / \sigma)(\partial \sigma / \partial T)=0.02 \mathrm{~K}^{-1}, \quad(1 / \varepsilon)(\partial \varepsilon / \partial T)=-4 \times 10^{-3} \mathrm{~K}^{-1}$, $\left(1 / \rho_{m}\right)\left(\partial \rho_{m} / \partial T\right)=-2 \times 10^{-4} \mathrm{~K}^{-1}, k=0.6 \mathrm{~J} \mathrm{~s}^{-1} \mathrm{~m}^{-1} \mathrm{~K}^{-1}$

Figure 1 shows the domains of influence of the different types of flows. The level curves represent the magnitude of the velocity field in meters per second (velocities below $0.01 \mu \mathrm{m} / \mathrm{s}$ are considered as undetectable and are represented by a white region in the diagrams). The first thing we note is that buoyancy dominates over all other types of flows for large systems. The second important effect is that ac electroosmosis is very important at low frequencies. Only for high fields the electroosmotic velocity is less than the electrothermal one. As we increase the frequency the ac electroosmosis decreases. Also as we increase the conductivity the Joule-heating induced flows, electrothermal and buoyancy, become dominant. 


\section{ACNOWLEDGMENTS}

This research has been supported by the Ministerio de Ciencia Y Tecnología del Gobierno Español, Dirección General de Investigación under project BFM200-1056.

\section{REFERENCES}

[1] R. Pethig, Crit. Revs. Biotech., 16 (1996) 331-348.

[2] T. Muller, A. Gerardino, T. Schnelle, S. G. Shirley, F. Bordoni, G. DeGasperis, R. Leoni, ànd G. Fuhr, J. Phys. D: Appl. Phys., 29 (1996) 340-349.

[3] A. Ramos, H. Morgan, N. G. Green, and A. Castellanos. J. Phys. D: Appl. Phys. 31:2338-2353 (1998)

[4] A. Ramos, H. Morgan, N.G. Green and A. Castellanos, J. Coll. Interf. Science, 217 (1999) 420-422.

[5] N.G. Green, A. Ramos, A. Gonzalez, H. Morgan and A. Castellanos, Phys. Rev. E, 61 (2000) 4011-4018.

[6] N. G. Green, A. Ramos, H. Morgan, A. Castellanos and A. González. J. Electrost: 53:71-87 (2001)

[7] A. Castellanos, Electrohydrodynamics: Chapter 4 (ed. A. Castellanos), Springer-Verlag, Wien, NewYork, 1998.

[8] A. González, A. Ramos, NG. Green, A. Castellanos and H. Morgan, Phys. Rev. E, 61, 4019-4028, (2000).

[9] NG. Green, A. Ramos, A. González, H. Morgan and A. Castellanos, Phys. Rev. E, (under revision) (2002)

\section{FIGURE 1}

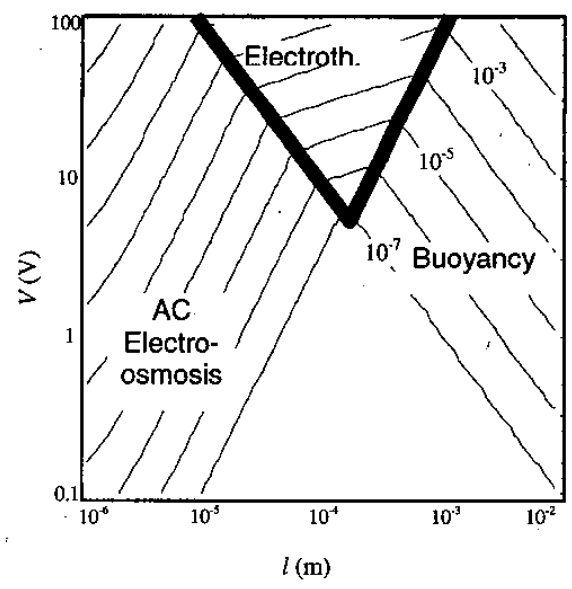

$\sigma=10^{-3} \mathrm{~S} / \mathrm{m}, f=1 \mathrm{kHz}$

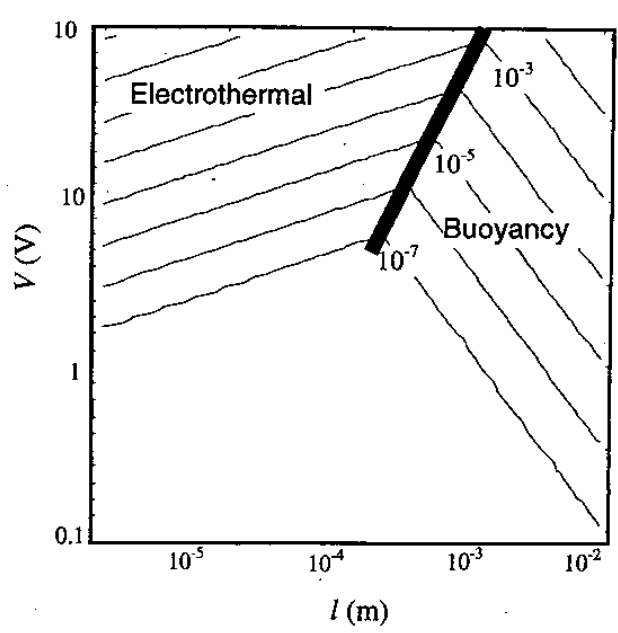

$\sigma=10^{-3} \mathrm{~S} / \mathrm{m}, f=1 \mathrm{MHz}$

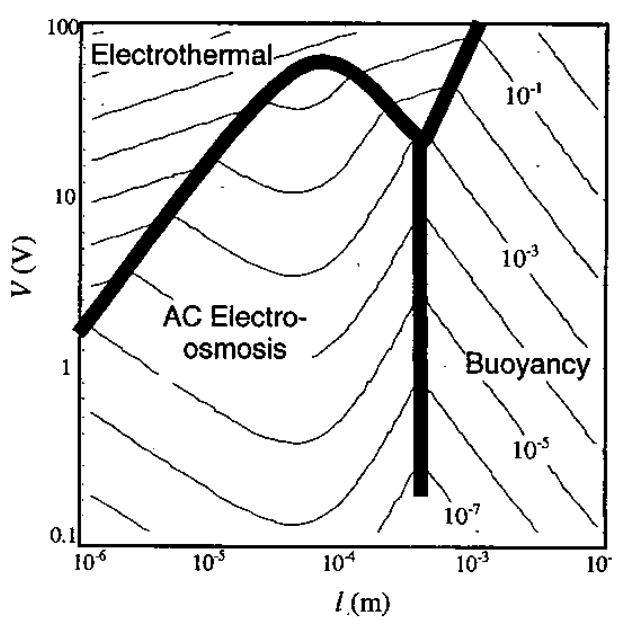

$\sigma=10^{-1} \mathrm{~S} / \mathrm{m}, f=1 \mathrm{kHz}$

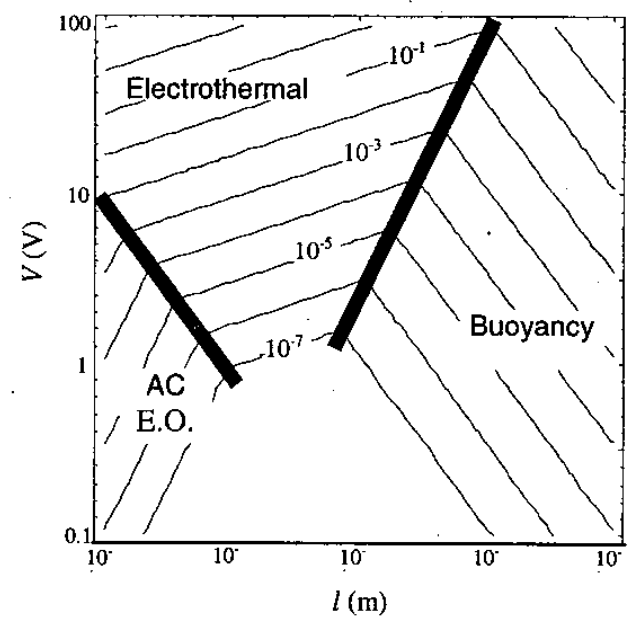

$\sigma=10^{-1} \mathrm{~S} / \mathrm{m}, f=1 \mathrm{MHz}$ 